\title{
Enhancement of Raman Scattering Efficiency by a Metallic Nano-antenna on Top of a High Index Contrast Waveguide
}

\author{
Frédéric Peyskens, Ananth Z. Subramanian, Ashim Dhakal, Nicolas Le Thomas and Roel Baets \\ Photonics Research Group, INTEC; Center for Nano- and BioPhotonics, Ghent University, 9000 Ghent, Belgium \\ frederic.peyskens@intec.ugent.be
}

\begin{abstract}
We theoretically study coupling of dipole radiation into integrated $\mathrm{Si}_{3} \mathrm{~N}_{4}$ strip waveguides functionalized with a nanoplasmonic antenna. This structure enables efficient coupling of enhanced Raman signals into the fundamental TE-mode of the waveguide.

OCIS codes: (130.0130) Integrated Optics; (250.5403) Plasmonics.
\end{abstract}

\section{Introduction}

The present lab-on-a-chip devices, which enable parallel study of multiple analytes, provide an alternative to current bulky technologies for spectroscopy and sensing. These technologies rely on labeling methods that are intrusive in nature. In this regard, integrated spectroscopic Raman sensors would enable label-free, high specificity and cheap sensing platforms. However, Raman signals are inherently very weak and therefore require an additional enhancement mechanism for efficient detection. Conventional Surface Enhanced Raman Spectroscopy (SERS) is based on the resonant plasmonic behavior of metallic nanoparticles or rough metallic surfaces to enhance these signals. Photonic integrated circuits offer the additional advantage of using single mode waveguides for excitation and collection of Raman signals in a more controlled way. CMOS-compatible photonic integration technologies are particularly relevant in this context since they hold the promise of low cost lab-on-a-chip devices. One can choose between silicon and silicon nitride $\left(\mathrm{Si}_{3} \mathrm{~N}_{4}\right)$ waveguides depending on the targeted spectral range.

It is known that high-index-contrast (HIC) waveguides and free-space nanoplasmonic antennas lead both to emission enhancement of dipoles [1-3]. In this paper we propose an approach towards combining a $\mathrm{HIC} \mathrm{Si}_{3} \mathrm{~N}_{4}$ strip waveguide and a nanoplasmonic antenna for enhanced Raman spectroscopy. A dipole is located in the surrounding of the nano-antenna, which lies on top of the waveguide, and is excited by the fundamental TE-mode $\left(\lambda_{0}=900 \mathrm{~nm}\right)$. We restrict ourselves to the TE-case since the TM polarization cannot efficiently excite the configuration under study. It is shown that the metallic nanostructure has no detrimental influence on the transmission of the dipole radiation. On the contrary, we report a transmission enhancement up to $10^{10}$ of hybrid $\mathrm{Si}_{3} \mathrm{~N}_{4}$-nanoplasmonics waveguides compared to simple $\mathrm{Si}_{3} \mathrm{~N}_{4}$ waveguides. The high transmission enhancement combined with the simple excitation and collection approach makes this platform a valuable candidate for single molecule SERS.

\section{Theoretical Investigation of Raman Enhancement}

In this study we consider a single mode $\mathrm{HIC} \mathrm{Si}_{3} \mathrm{~N}_{4}$ strip waveguide $(\mathrm{n}=2.02$, width $=600 \mathrm{~nm}$ and height $=220 \mathrm{~nm})$ on a $\mathrm{SiO}_{2}$ substrate $(\mathrm{n}=1.45)$ and water cladding $(\mathrm{n}=1.33)$ with a gold bowtie antenna on top (Fig. 1(a)). This shape is chosen since strong fields are expected at the tips of the antenna, which is important if we target single molecule SERS. By means of the commercial finite element solver COMSOL the interaction between the guided mode of the waveguide and a dipole is investigated for two cases: a dipole in the gap of the bowtie antenna and a dipole on the same waveguide but without the antenna (Fig. 1(a)). The dipole power coupled to the waveguide mode is denoted by $P_{e n h}$ and $P_{0}$ for the cases with and without the gold, respectively. The enhancement factor EF is defined as $P_{e n h} / P_{0}$. In order to test the meshing quality and the numerical calculation parameters, the total radiated power for a dipole parallel and perpendicular to the core region of a slab waveguide is calculated. An excellent correspondence (average error $\sim 2 \%$ ) was found between our numerical results and the exact analytical results [4]. So the EFs discussed hereafter are expected to have a high accuracy. Since the fundamental TE-mode (polarized along $\boldsymbol{e}_{\boldsymbol{x}}$ ) is used as the excitation field, the dominant and relevant part of the Raman dipole moment is given by $p_{x}\left(\boldsymbol{r}_{0}\right)=\alpha_{x x} E_{x}\left(\boldsymbol{r}_{0}\right)$; $\alpha_{x x}$ is the $(1,1)$ component of the polarizability tensor given by the Kramers-Heisenberg-Dirac formula [5]. Only positions in the gap of the gold structure are considered since maximum EFs are expected there. In Fig. 1(b) we plot the ratio $P_{e n h} / P_{0}$ as a function of the ratio $\beta=\left|E_{e n h}\left(\boldsymbol{r}_{0}\right)\right| \lambda E_{0}\left(\boldsymbol{r}_{0}\right) \mid$ (each dot corresponds to a different position $\boldsymbol{r}_{0}$ of the dipole); $E_{\text {enh }}\left(\boldsymbol{r}_{0}\right)$ is the enhanced excitation field due to the plasmon resonance and $E_{0}\left(\boldsymbol{r}_{0}\right)$ is the evanescent field of the regular waveguide. It is clear that the simulation results (red dots) match the fitted $\beta^{4}$ curve (with a given scaling factor that will depend on the specific configuration and wavelength). Since the excitation enhancement scales with $\beta^{2}$ (because the respective dipole moments scale with $\beta$ ), it follows from this figure that the transmitted power by the dipole also scales with $\beta^{2}$. Addition of a metallic nanostructure on top of a HIC waveguide has thus no quenching 
effect on the transmitted power by the dipole into the guided mode. So there is an efficient coupling of the enhanced emitted power into the fundamental TE-mode of the waveguide. From the above observations, three distinct advantages of using integrated waveguides with a nanoplasmonic structure emerge. Firstly, the plasmons are always excited with the proper polarization to ensure optimal excitation enhancement. Secondly, the nanoplasmonic structure has no detrimental impact on the output power but enables a significant emission enhancement compared to a regular $\mathrm{HIC} \mathrm{Si}_{3} \mathrm{~N}_{4}$ waveguide (see Fig. 1(b)). Finally, the emitted signal is coupled efficiently to the fundamental mode of the waveguide. This creates ideal conditions for on-chip spectral analysis of the signal. It is important to note that the absolute value of $P_{e n h}$ depends on both the excitation and emission wavelength. For small Stokes shifts, the excitation and emission enhancement factors will be practically equal as the resonance of the combined waveguide-plasmonics system is quite broadband, but for larger shifts the emission enhancement of $P_{e n h}$ (and thus the actual output power) will decrease.
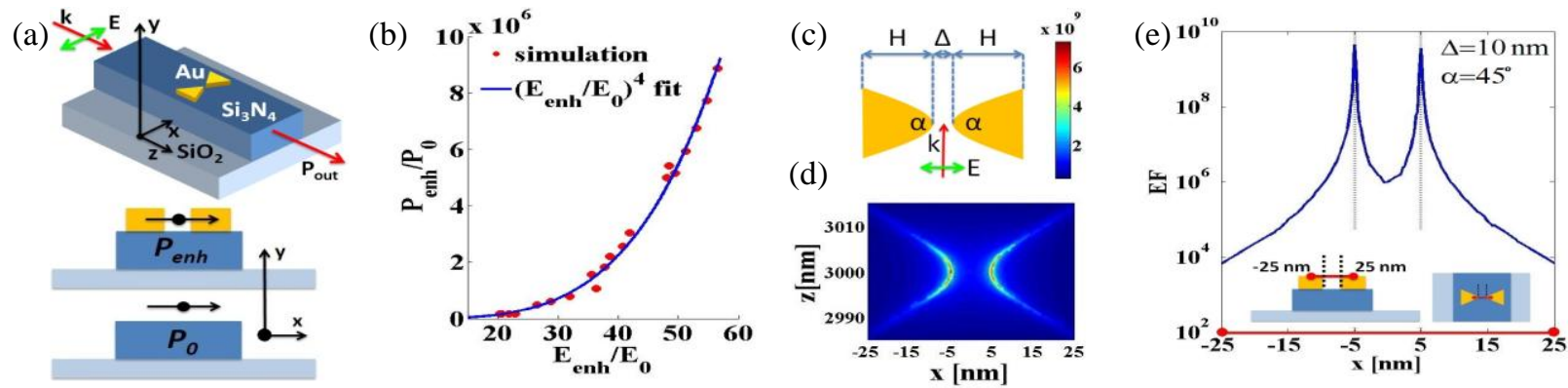

Fig. 1. (a) $\mathrm{HIC} \mathrm{Si}_{3} \mathrm{~N}_{4}$ strip waveguide functionalized with a bowtie antenna; the polarization (green double arrow) and propagation (red arrow) direction of the modal field and the orientation of the radiating dipole are also marked. (b) EF of the Raman power coupled into the guided mode as a function of the field enhancement. (c) Bowtie antenna with dimensions $(H, \Delta, \alpha)$. (d) Electric field distribution at the top surface of the gold for $\alpha=60^{\circ}$ and $\Delta=10 \mathrm{~nm}$. (e) $\mathrm{EF}$ at the top surface of the gold; the two inset figures mark the $(y, z)$-position where the slice (red line) is taken.

\section{Parameter study of the bowtie antenna}

In this section we investigate the impact of the parameters of the bowtie antenna (Fig. 1(c)) to study how we can optimize the excitation and emission of a dipole. EFs for different geometry parameters are calculated via $\beta^{4}$ since Fig. 1(b) shows that EF scales with $\beta^{4}$. Figure 1(d) gives an example of the field distribution in the gap of the antenna. For a dipole in the center of the gap there is an optimal angle $\alpha\left(\right.$ here $\sim 60^{\circ}$ ) which maximizes the EF. For a dipole at the tip, smaller angles are better for larger EFs. Generally, the optimal height for a given angle is dependent on the gap and for a given gap the optimal height depends on the angle. In the center of the gap there is a $10^{3}$ order difference for $\Delta=10 \mathrm{~nm}$ and $\Delta=40 \mathrm{~nm}$ and changing $H$ by only $20 \mathrm{~nm}$ from the optimal $H$ reduces the EF by 10 . The $\mathrm{EF}$ at the tips decreases by 2 orders if we change $\alpha$ from $30^{\circ}$ to $90^{\circ}$ (for a fixed gap). So, depending on the position of the dipole the $\mathrm{EF}$ is maximized for specific values of the $(H, A, \alpha)$ triplet. Figure 1(e) shows the EF at the top surface (and in the center) of the gold structure. The strong enhancement at the tips and the steep decay of the EF are clearly visible. Our study shows that for $\alpha=30^{\circ}$ and $\Delta=10 \mathrm{~nm}$ it is possible to obtain transmission enhancements EF up to $10^{10}$ in a $1 \mathrm{~nm}$ region near the tip (the mesh-size near the tip is $1 \mathrm{~nm}$ ). As such it is clear that adding a properly designed nanoplasmonic structure gives an additional gain up to $10^{10}$ compared to the enhancement that a HIC waveguide has. This controllable high EF opens the possibility of doing single molecule SERS with our investigated structure. Currently, fabrication of such gold nanoplasmonic structures is explored by means of Focused Ion Beam (FIB) milling and e-beam lithography on top of a $\mathrm{Si}_{3} \mathrm{~N}_{4}$ waveguide circuit processed in a CMOS fab.

\section{Conclusion}

In this paper, it is established that the radiation originating from TE-excited dipoles is coupled efficiently into the fundamental TE-mode of a $\mathrm{Si}_{3} \mathrm{~N}_{4}$ strip waveguide. We also showed that adding a single, well-designed, nanoplasmonic structure has no negative impact on the transmission of the coupled dipole radiation, but is capable of producing Raman scattering enhancements of $10^{10}$ compared to a simple $\mathrm{Si}_{3} \mathrm{~N}_{4}$ strip waveguide.

\section{References}

[1] Y.C. Jun, et al., "Broadband enhancement of light emission in silicon slot waveguides," Optics Express 17(9), 7479-7490 (2009).

[2] K.C. Y. Huang, et al., "Power flow from a dipole emitter near an optical antenna," Optics Express 19(20), 19084-19092 (2011).

[3] P.A. Gonzalez, et al., "Resolving the electromagnetic mechanism of surface-enhanced light scattering at single hot spots," Nature Communications 3, 1-7 (2012).

[4] L. Novotny and B. Hecht, Principles of Nano-Optics (Cambridge University Press, 2006), Chap. 10.

[5] J.R. Lombardi and R.L. Birke, “A Unified Approach to Surface-Enhanced Raman Spectroscopy,” J.Phys.Chem. C 112, $5605-5617$ (2008). 